\title{
The location of energetic compartments affects energetic communication in cardiomyocytes
}

\author{
Rikke Birkedal *, Martin Laasmaa and Marko Vendelin \\ Laboratory of Systems Biology, Institute of Cybernetics, Tallinn University of Technology, Tallinn, Estonia
}

\section{Edited by:}

Miguel A. Aon, Johns Hopkins

University School of Medicine, USA

Reviewed by:

Nazareno Paolocci, Johns Hopkins University, USA

Tatiana Rostovtseva, National

Institutes of Health, USA

*Correspondence:

Rikke Birkedal, Laboratory of

Systems Biology, Institute of Cybernetics, Tallinn University of

Technology, Akadeemia 21,

12618 Tallinn, Estonia

e-mail: rikke@sysbio.ioc.ee
The heart relies on accurate regulation of mitochondrial energy supply to match energy demand. The main regulators are $\mathrm{Ca}^{2+}$ and feedback of $\mathrm{ADP}$ and $\mathrm{P}_{\mathrm{i}}$. Regulation via feedback has intrigued for decades. First, the heart exhibits a remarkable metabolic stability. Second, diffusion of ADP and other molecules is restricted specifically in heart and red muscle, where a fast feedback is needed the most. To explain the regulation by feedback, compartmentalization must be taken into account. Experiments and theoretical approaches suggest that cardiomyocyte energetic compartmentalization is elaborate with barriers obstructing diffusion in the cytosol and at the level of the mitochondrial outer membrane (MOM). A recent study suggests the barriers are organized in a lattice with dimensions in agreement with those of intracellular structures. Here, we discuss the possible location of these barriers. The more plausible scenario includes a barrier at the level of MOM. Much research has focused on how the permeability of MOM itself is regulated, and the importance of the creatine kinase system to facilitate energetic communication. We hypothesize that at least part of the diffusion restriction at the MOM level is not by MOM itself, but due to the close physical association between the sarcoplasmic reticulum (SR) and mitochondria. This will explain why animals with a disabled creatine kinase system exhibit rather mild phenotype modifications. Mitochondria are hubs of energetics, but also ROS production and signaling. The close association between SR and mitochondria may form a diffusion barrier to ADP added outside a permeabilized cardiomyocyte. But in vivo, it is the structural basis for the mitochondrial-SR coupling that is crucial for the regulation of mitochondrial $\mathrm{Ca}^{2+}$-transients to regulate energetics, and for avoiding $\mathrm{Ca}^{2+}$-overload and irreversible opening of the mitochondrial permeability transition pore.

Keywords: ADP, calcium, cardiomyocytes, creatine kinase, energetic compartments, mitochondria, oxidative phosphorylation, regulation

\section{REGULATION OF MITOCHONDRIAL ENERGY PRODUCTION IN CARDIOMYOCYTES}

The heart can never rest. During high workloads, skeletal muscle may develop an "energy debt," which is paid back during rest. But the heart must avoid such an energy debt, as it cannot take a few minutes off to rest and restore its energy levels. Therefore, it is crucial to quickly and precisely regulate energy generation to match the energy consumption in time and space. The primary energy source in the heart is mitochondrial oxidative phosphorylation, which is mainly regulated by $\mathrm{Ca}^{2+}$ and $\mathrm{ADP} / \mathrm{P}_{\mathrm{i}}$-feedback.

$\mathrm{Ca}^{2+}$ exhibits "parallel regulation" of myofibrillar contraction and mitochondrial energy supply. $\mathrm{Ca}^{2+}$ enters the mitochondria through the $\mathrm{Ca}^{2+}$ uniporter, and is pumped out again mainly via the mitochondrial $\mathrm{Na}^{+} / \mathrm{Ca}^{2+}$-exchanger (Wei et al., 2011). This happens on a beat-to-beat basis. Mitochondrial $\mathrm{Ca}^{2+}$-transients have the same time to peak as cytosolic $\mathrm{Ca}^{2+}$-transients, but slower decay ( $\mathrm{Lu}$ et al., 2013). $\mathrm{Ca}^{2+}$ in the mitochondrial matrix stimulates pyruvate, isocitrate and $\alpha$-ketoglutarate dehydrogenase (McCormack et al., 1990), which reduce NAD to NADH in the citric acid cycle, as well as the $\mathrm{F}_{1} \mathrm{~F}_{0}$-ATPase (Territo et al., 2000). Mitochondrial $\mathrm{Ca}^{2+}$-uptake increases with contraction frequency and adrenergic stimulation (Lu et al., 2013). Thus, as the $\mathrm{Ca}^{2+}$. transient increases to make cardiomyocytes contract faster and with greater force, so is the mitochondrial $\mathrm{Ca}^{2+}$-uptake enhanced to further stimulate mitochondrial energy generation.

$\mathrm{ADP}$ and $\mathrm{P}_{\mathrm{i}}$, on the other hand, exhibit "feedback regulation" of mitochondrial energy supply. In contrast to $\mathrm{Ca}^{2+}$, which has a steep electrochemical gradient and is let into the cytosol and pumped out, the feedback regulation depends on the energetic circuit, where ATP diffuses from the mitochondria to the ATPases, and ADP and $\mathrm{P}_{\mathrm{i}}$ diffuse from the ATPases to the mitochondria. The importance of feedback as a regulator of mitochondrial energy supply is intriguing, because the heart exhibits a remarkable metabolic stability: the ADP-concentration is unchanged even during large increases in workload and oxygen consumption (Katz et al., 1989; Balaban, 2002). $\mathrm{P}_{\mathrm{i}}$-concentration changes the most, and some studies suggest $\mathrm{P}_{\mathrm{i}}$ to be an important regulator, in particular in low to moderate workloads (Saks et al., 2000; Bose et al., 2003; Wu et al., 2008). Irrespectively, it is intriguing 
that specifically in cardiomyocytes and red muscle, where feedback regulation is needed the most, there seems to be barriers that obstruct diffusion significantly.

In working heart trabeculae, NADH fluorescence decreases and then partially recovers upon an increase in work (Brandes and Bers, 2002). This suggests that there are multiple regulators of mitochondrial energy production. As noted above, $\mathrm{Ca}^{2+}$ stimulates dehydrogenases to produce NADH. It also stimulates $\mathrm{F}_{1} \mathrm{~F}_{\mathrm{o}}$-ATPase. But overall, $\mathrm{Ca}^{2+}$-uptake by isolated mitochondria leads to an increase in NADH (Territo et al., 2001). NADH increases or decreases with $\mathrm{P}_{\mathrm{i}}$ depending on the presence of ADP (Bose et al., 2003). ADP stimulates respiration rate, which decreases NADH fluorescence and increases flavoprotein fluorescence (Jepihhina et al., 2011). Cortassa and collaborators made an integrated model taking into account how excitation contraction coupling influences mitochondrial energetics (Cortassa et al., 2006). With this model, they were able to reproduce the experimental data of Brandes and Bers (2002) and analyze the regulatory mechanisms. Their quantitative analysis suggests that during work transitions energy supply is regulated initially by feedback, which decreases NADH. Subsequent parallel regulation by $\mathrm{Ca}^{2+}$ counterbalances this decrease, and NADH recovers (Cortassa et al., 2006). To explain how feedback can respond so quickly and be so important, despite overall metabolic stability, it is necessary to take into account energetic compartmentalization in cardiomyocytes.

It is well recognized that $\mathrm{Ca}^{2+}$ compartments exist in cardiomyocytes. Local $\mathrm{Ca}^{2+}$-events are visible with $\mathrm{Ca}^{2+}$-indicators (Wang et al., 2004). Due to the low $\mathrm{Ca}^{2+}$-affinity of the mitochondrial $\mathrm{Ca}^{2+}$-uniporter, mitochondrial $\mathrm{Ca}^{2+}$-uptake would not take place if it were not for the structural proximity between mitochondria and the sarcoplasmic reticulum (SR) (FranziniArmstrong, 2007). There is, however, no direct coupling, and $\mathrm{Ca}^{2+}$ has to diffuse from the SR to the mitochondria (FranziniArmstrong, 2007). This leads to an intra-mitochondrial $\mathrm{Ca}^{2+}$. gradient (Lu et al., 2013). The other major signaling molecule in the cell, cyclic AMP, is also confined to compartments. This is the only way to explain that stimulation of specific receptors using the same signaling cascade components ( $G_{s}$ proteins, cyclic AMP, and protein kinase A) leads to specific responses (Kritzer et al., 2012; Mika et al., 2012).

With these considerations in mind, it is not so surprising that cardiomyocytes also have energetic compartments with local concentrations of ADP, $\mathrm{P}_{\mathrm{i}}$, and ATP. Actually, energetic compartmentalization of cardiac tissue was suggested already in 1970, where Gudbjarnason et al. showed that after induction of ischemia, cardiac contraction declines with the concentration of phosphocreatine $(\mathrm{PCr})$, while overall ATP remains unchanged (Gudbjarnason et al., 1970). It is difficult to assess energetic compartments as there are no good fluorescent indicators for ADP, $\mathrm{P}_{\mathrm{i}}$, and ATP (as for $\mathrm{Ca}^{2+}$ ). As an indirect measure, many studies of energetic compartmentalization have assessed and/or modeled mitochondrial function in permeabilized fibers and cardiomyocytes - traditionally during ADP- and ATP-titrations. Mitochondria in permeabilized cardiomyocytes are characterized by an apparent ADP-affinity that is much lower than that of isolated mitochondria. This is taken to indicate significant diffusion restriction between the medium outside the permeabilized cell and the adenine nucleotide translocase (ANT) in the mitochondrial inner membrane. Questions that are still being debated are: What causes the restriction of diffusion? And how does it affect energetic communication between ATPases and mitochondria via feedback?

The exact location of energetic compartments may differ from other molecules compartments, but the basic principles of compartment formation are the same. The concentration of a molecule in a given location depends on (1) the reaction rate and relative location of proteins involved in its production/release and consumption/uptake, (2) its diffusion speed, which in turn depends (among other things) on its concentration gradient, (3) its buffering by particulate and/or soluble proteins in the cell, and (4) the organization of physical barriers in the form of membrane structures, organelles, and macromolecular complexes, which may obstruct diffusion. If the sites of synthesis and consumption are close to each other, the molecule may be immediately consumed thus not being able to diffuse to other parts of the cell. Thus, the molecule concentration will be locally much higher compared to the bulk, and the enzymes processing the molecule are said to be coupled. This seems to be the mechanism regulating the compartmentalization of cyclic AMP (Kritzer et al., 2012; Mika et al., 2012). Any enzyme pair with common substrate/product can become coupled. One prerequisite is, however, that they are bound in each other's vicinity, so that the substrate/product is channeled between the enzymes within the unstirred layer immediately above the surface (Goldman and Katchalski, 1971; Arrio-Dupont et al., 1985; Fossel and Hoefeler, 1987; Arrio-Dupont, 1988). If the molecule is consumed further away from its generation site, it has enough time to diffuse. Then, its distribution in the cell depends more on diffusion speed, buffering, and physical structures forming diffusion barriers. Much of molecular motion in the cell occurs by diffusion (Kinsey et al., 2011). For example, diffusion of ROS is effective in the micrometer range, making it a feasible mechanism of communication between mitochondria (Aon et al., 2004).

\section{ENERGETIC COMPARTMENTS IN DIFFERENT SIZES}

Experimental data suggest there to be multiple energetic compartments scaling in size from coupled enzymes to the proposed intracellular energetic units (ICEUs) (Saks et al., 2001). Starting with the smallest, good examples of coupled enzyme pairs are those of creatine kinase (CK), which is bound near and coupled to various cellular ATPases such as myosin ATPase (Ventura-Clapier et al., 1987; Arrio-Dupont, 1988; Haagensen et al., 2008), the sarco-endoplasmic reticulum $\mathrm{Ca}^{2+}$-ATPase, i.e., SERCA (Minajeva et al., 1996), the $\mathrm{Na}^{+} / \mathrm{K}^{+}$ATPase (Grosse et al., 1980), and the $\mathrm{K}_{\mathrm{ATP}}$-channel (Crawford et al., 2002). Here, the ATPases hydrolyze ATP to ADP and $\mathrm{P}_{\mathrm{i}}$, and CK uses PCr to regenerate ADP to ATP. In the mitochondria, the reaction goes the other way: ADP is phosphorylated to ATP, and the mitochondrial form of CK (Mi-CK) in the inter-membrane space uses creatine to regenerate ADP (Wallimann et al., 1992). Structural and model studies have confirmed that Mi-CK is bound near the ANT via its binding to cardiolipin in the inner mitochondrial membrane (Rojo et al., 1991; Schlattner et al., 2009; 
Karo et al., 2012), suggesting direct metabolite transfer between them (Vendelin et al., 2004b). The reaction may also go in the direction of ADP and PCr synthesis in the cytosol, where CK may be coupled to glycolytic enzymes (Kraft et al., 2000).

Larger compartments depend more on physical structures forming diffusion barriers. At the next size level, compartments are represented by organelles. In cardiac tissue, mitochondria are the organelles taking up the largest volume, 20-30\%, whereas next in size the SR represents $4.5 \%$ of the cell volume (Decker et al., 1991). In mitochondria, the double membrane results in an inter-membrane as well as a matrix compartment. In cardiomyocytes, there is a significant barrier for ADP at the level of the mitochondrial outer membrane (MOM). This was first suggested based on the apparent ADP-affinity being much lower in permeabilized fibers than in isolated mitochondria (Saks et al., 1991, 1993; Kuznetsov et al., 1996). Indeed, adenine nucleotides pass the MOM through the voltage gated anion channel (VDAC) (Rostovtseva and Colombini, 1997), and the permeability of VDAC can be regulated by tubulin (Rostovtseva et al., 2008; Rostovtseva and Bezrukov, 2012).

In cardiomyocytes, even larger compartments have been proposed to exist. They were named ICEUs (Saks et al., 2001). Although their delimiters are still not identified, it has been suggested that t-tubules, organelles, and macromolecular complexes are organized in such a manner that ATPases are grouped with mitochondria. The existence of ICEUs was proposed on the basis of experiments suggesting the existence of cytoplasmic diffusion restrictions. Diffusion restriction by MOM can explain the much lower ADP-affinity in permeabilized cardiomyocytes. However, it cannot explain the fact that ADP and $\mathrm{P}_{\mathrm{i}}$ generated inside permeabilized cardiomyocytes seem to be "channeled" to the mitochondria rather than out of the solution (Kummel, 1988; Seppet et al., 2001). For this, there has to be cytosolic diffusion restrictions as well. Modeling shows that these are localized rather than uniformly distributed in the cytoplasm (Vendelin et al., 2004a). A more elaborate 3D model shows a possible arrangement of the diffusion restrictions, which are at the level of MOM and as sheets between mitochondria-probably formed by the SR and cytoskeletal proteins (Ramay and Vendelin, 2009).

\section{ENERGETIC COMPARTMENTS AFFECT ENERGETIC COMMUNICATION-BUT HOW?}

In the energetic circuit between ATPases and mitochondria, the main issue is how to efficiently transport ADP and $\mathrm{P}_{\mathrm{i}}$ from ATPases to the mitochondria. This ensures an adequate phophorylation potential near ATPases and regulation of mitochondrial energy production. Overall, ADP ranges in the $\mu \mathrm{M}$, whereas ATP, $\mathrm{P}_{\mathrm{i}}, \mathrm{PCr}$, and creatine range in the $\mathrm{mM}$ (Wallimann et al., 1992). $\mathrm{P}_{\mathrm{i}}$-concentration varies the most with changes in work (Wu et al., 2008). However, due to its low concentration, even small changes in ADP have a large effect on the phosphorylation potential, which must be above a certain value for ATPases to obtain sufficient energy from ATP hydrolysis.

The physiological importance of coupled enzymes is quite obvious. The functional coupling between cytosolic CK and ATPases is beneficial when energy demand exceeds energy supply, and PCr is used to buffer the ADP/ATP-ratio and thus the phosphorylation potential. This situation has been dubbed "temporal energy buffering," that is characterized by a net consumption of PCr to buffer ATP. In cardiomyocytes, the functional coupling between Mi-CK and ANT is beneficial, because oxidative phosphorylation is the main source of energy, which can be stored as PCr. The CK equilibrium constant favors ADP phosphorylation. Thus, locally high ATP concentrations or direct transfer of ATP from ANT is needed for Mi-CK to generate PCr. The same is true for cytoplasmic CK coupled to glycolytic enzymes.

On a slightly larger scale, the situation becomes more debatable. In addition to the temporal energy buffering, CK has been suggested to function as a spatial buffer. The spatial buffering occurs because the $\mathrm{CK}$ system forms an energy circuit with creatine and PCr, which runs in parallel with that of ADP and ATP. In the presence of spatial energy buffering there is no net consumption of high-energy phosphates, i.e., consumption matches generation. Thus, the CK system or "CK shuttle" facilitates energetic communication between ATPases and ATP-producing sites.

In the heart that depends on reliable regulation of mitochondrial energy, the CK system has been assumed by other investigators to be paramount. Indeed, it seems paradoxical that in oxidative muscles, that rely on energy generated by mitochondria, there is a significant barrier obstructing the feedback from ATPases at the MOM level (Kuznetsov et al., 1996; VenturaClapier et al., 1998). As an explanation, it has been proposed that the MOM permeability is regulated to ensure energetic communication via the CK system (Saks et al., 1994). While this proposal is appealing, the role of the CK system continues to be debated. As noted above, it has been shown that dimeric tubulin binds VDAC and can restrict its permeability (Rostovtseva and Bezrukov, 2012). But it has yet to be established whether such a restriction actually occurs in vivo and whether this restriction is regulated in the heart. If such regulation occurs, we would expect $\mathrm{MOM}$ to be more permeable in the absence of a functional CK system.

Experiments where the CK system was inhibited by feeding with beta-guanidinoproionic acid (a creatine analog) or knockout of one or more CK isoforms have shown varying effects on cardiac function. In general, the hearts seem to adapt to cope with basal workloads, but they fail under high workload conditions, fast work transitions and ischemia (Shoubridge et al., 1985; Mekhfi et al., 1990; Zweier et al., 1991; Neubauer et al., 1999; Kaasik et al., 2001; Crozatier et al., 2002; Spindler et al., 2004; Nahrendorf et al., 2005). In studies of knockout mice, genetic background has turned out to be important, and backcrossing seems to result in a milder phenotype (Lygate et al., 2009, 2012). It has been surprising that, so far, no compensatory changes have been found in heart of mice lacking guanidineacetate methyltransferase (GAMT; an enzyme in the creatine synthesis pathway), where the CK system is non-functional due to lack of creatine. These mice are smaller in size (Schmidt et al., 2004) but exhibit the same exercise capacity and tolerance to chronic myocardial infarction of their wild type littermates (Lygate et al., 2013). Furthermore, in a follow up study it was shown that intracellular compartmentalization as well as diffusion across MOM and mitochondrial organization were unchanged (Branovets et al., 2013). GAMT deficient mice accumulate guanidinoacetate, 
which is phosphorylated and may be used instead of creatine by CK in critical situations (Boehm et al., 1996; Kan et al., 2004). However, guanidinoacetate is not used by Mi-CK (Boehm et al., 1996), thus being unable to facilitate transport across MOM. Consequently, the cardiomyocytes of these mice do relatively well without a CK system, despite the fact that diffusion is restricted at the level of MOM to the same extent as in wildtype. This is in agreement with the idea that mitochondria are able to supply energy for SERCA-mediated $\mathrm{Ca}^{2+}$-uptake with the same efficiency as CK (Kaasik et al., 2001). However, this interpretation raises the following question: is the $\mathrm{CK}$ system needed to facilitate ADP/ATP transport across MOM in the heart? At present, we have no firm answer to this question, and further studies are needed.

It must be noted that spatial energy buffering by $\mathrm{CK}$ may not exclusively mean that CK facilitates energetic communication across MOM. Experiments with mice overexpressing M-CK showed that the higher M-CK activity and the associated higher CK flux significantly improves cardiac function after ischemiareperfusion (Akki et al., 2012) and in failing hearts (Gupta et al., 2012). Indeed, the decrease in CK flux can be used as a predictor of heart failure (Bottomley et al., 2013). Taken together these data indicate that lack of CK does not worsen heart failure and that M-CK overexpression has therapeutic potential (Lygate and Neubauer, 2014). Whereas the rescue by M-CK overexpression seems to be at odds with the lack of changes in knockout models, differences in metabolism and energy transfer during energy starvation might provide an explanation. In the healthy heart, $\mathrm{CK}$ activity is moderate and a significant fraction is accounted for by Mi-CK. In glycolytic muscles, the total CK activity is higher than in oxidative muscle, mainly due to cytosolic M-CK (Ventura-Clapier et al., 1998). Both ischemic and failing hearts exhibit insufficient mitochondrial energy generation, relying on glycolytic energy supply to a large extent. This prompts us to ask whether hearts overexpressing $\mathrm{M}-\mathrm{CK}$ are rescued because of a higher M-CK activity enabling a more efficient energy transfer between glycolytic enzymes and ATPases.

The importance of CK as a spatial energy buffer has also been studied using theoretical approaches. Some of these studies support the idea that CK-mediated enhancement of the ADP feedback is important (Wu and Beard, 2009). A model based on NMR data suggested that under normal conditions, energetic communication may occur via direct ADP/ATP transport as well as creatine/PCr transport. In contrast, at high workload, the CK system is bypassed (Vendelin et al., 2010). A recent study shows that energy transport via the CK system amounts to no more than 15\% (Hettling and van Beek, 2011). In fact, temporal and spatial energy buffering by the CK system are inseparable (Meyer et al., 1984). This is in agreement with the idea that the mitochondrial response to a change in heart rate is faster in CK knockout mice (Gustafson and van Beek, 2002). In view of these data, we ask whether in the heart, spatial energy buffering is needed under physiological conditions, and for facilitated transport across MOM.

Model simulations of kinetic data suggest moderate diffusion restriction at the MOM level, but not necessarily by MOM itself (Ramay and Vendelin, 2009; Sepp et al., 2010). Thus, a significant part of the low apparent ADP-affinity in permeabilized cells is due to cytoplasmic diffusion restrictions forming ICEUs. ICEUs explain why ADP is channeled to mitochondria rather than out of the cell (Seppet et al., 2001), and why mitochondrial ATP is as efficient as CK in providing ATP to SR and myofilaments with ATP (Kaasik et al., 2001). Hypothetically, ICEUs confine mitochondria and ATPases in smaller compartments to reduce diffusion distances and ensure direct energetic communication. However, it remains an open question whether the ICEUs are "designed" in the sense that some structures are specifically organized in order to form ICEUs, or whether ICEUs are simply the consequence of cellular organization.

Figure 1A shows the regular arrangement of mitochondria in a cardiomyocyte. Figure 1B displays the probability distribution of neighboring mitochondria around a central mitochondrion. Of note is the circular arrangement of mitochondria in the crosssection (Birkedal et al., 2006). Does this arrangement explain energetic coupling between mitochondria and ATPases? Rows of myofilaments are surrounded by rows of mitochondria that function as sinks for ADP and Pi. Oppositely, rows of mitochondria are also surrounded by rows of myofilaments that function as sinks for ATP. It seems natural that in a feedback system with energetic circuits, the majority of metabolites diffuse down their concentration gradients the shortest possible path rather than diffusing out of the permeabilized cell.
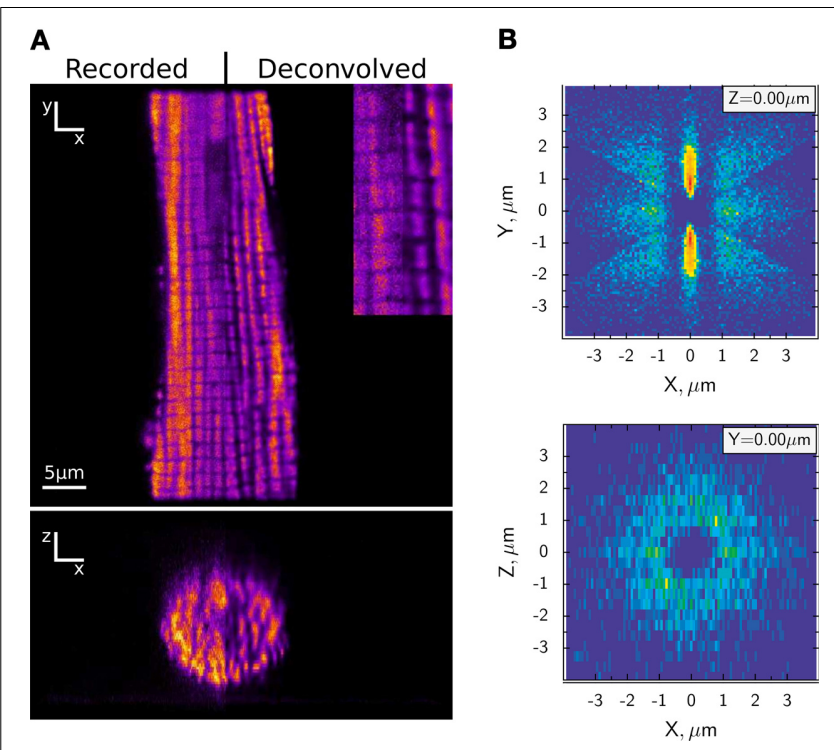

FIGURE 1 | Highly ordered arrangement of intermyofibrillar mitochondria in rat cardiomyocytes. (A) Representative confocal image of MitoTracker Green labeled mitochondria are shown on the top (XY) and reconstructed cross-section $(X Z)$ at the bottom. The original images are compared with deconvolved images after applying algorithms developed in Laasmaa et al. (2011). (B) Probability density of the closest mitochondrial centers in each sector of a rat cardiomyocyte, calculated as described in Birkedal et al. (2006). The density is shown in pseudo color with blue corresponding to regions where no neighboring mitochondria were found and red to the regions with high probability of finding the center of neighboring mitochondria. Note that mitochondria are arranged in a regular pattern (XY plane) with parallel rows separated by $\sim 1.8 \mu \mathrm{m}$ that can be found in any transversal direction relative to each other ( $X Z$ plane). For details of the analysis, see Birkedal et al. (2006). 
In the quest for the location of cytosolic diffusion restrictions, we have studied intracellular diffusion using raster image correlation spectroscopy (RICS). We first showed that radial diffusion of fluorescently labeled ATP in cardiomyocytes is slower than transversal diffusion (Vendelin and Birkedal, 2008). A later study used two fluorescent molecules of different size (Illaste et al., 2012). Here, it was intriguing to find that in the cardiomyocyte compared to solution, diffusion of the large molecule was less restricted than diffusion of the small molecule. A stochastic model predicted that diffusion restrictions form a lattice with dimensions that are in agreement with the cardiomyocyte ultrastructure (Illaste et al., 2012). In Figure 2, we take a step further and draw this lattice superimposed on the cardiomyocytes ultrastructure.

Two possible scenarios are represented in Figure 2. In the first one (Figure 2A), the lattice is superimposed onto the mitochondrial membranes and the $\mathrm{m}$-band and $\mathrm{z}$-line of the sarcomere. In the second one (Figure 2B), half of a mitochondrion is grouped together with a fraction of a sarcomere. Considering the structures in the cell, the former seems more plausible: Transversally, diffusion barriers are formed by mitochondrial and SR membranes whereas longitudinal barriers are constituted by proteindense regions in the myofilaments ( $\mathrm{z}$-lines and $\mathrm{m}$-bands) and probably with some help from the junctional SR and t-tubules. However, the problem is that diffusion restriction at the level of MOM seems to separate rather than group together mitochondrial energy generation and ATPase energy consumption. An alternative explanation would be that diffusional restrictions at MOM are not due to the membrane itself but a result of its close association with the SR. Much of the work on energetic compartmentalization uses permeabilized cardiomyocytes, which are kept in a relaxed state. Although non-physiological, this is a useful preparation since it represents a simple situation. However, the importance of the SR is difficult to study in permeabilized cardiomyocytes, as SERCA is not active in this preparation (Sepp et al., 2014). As a membranous structure, the SR associated with the mitochondria will restrict diffusion from the medium to mitochondria in permeabilized cardiomyocytes. However, it also forms the structural basis for the energetic coupling between SERCA and mitochondria (Kaasik et al., 2001).
A fully developed cardiac CK system with a relatively high expression of mitochondrial as well as cytosolic CK isoforms is mainly found in adult mammals. The fact that mitochondrial CK seems absent in the hearts of lower vertebrates such as fish and frog can be explained by that they have a lower body temperature and cardiac performance, and depend more on glycolytic energy production (Ventura-Clapier et al., 1998; Birkedal and Gesser, 2006; Sokolova et al., 2009). Likewise, neonatal mammals with lower cardiac performance and higher reliance on glycolysis do not express Mi-CK (Hoerter et al., 1991, 1994; Tiivel et al., 2000). As the cardiomyocytes mature, they increase in diameter and develop from a relatively simple morphology to multiple parallel rows of myofibrils and mitochondria organized in a crystal-like pattern as we know it in adult cardiomyocytes (Vendelin et al., 2005; Birkedal et al., 2006; Anmann et al., 2014). In parallel, they develop t-tubules and a more elaborate SR (Sedarat et al., 2000; Dan et al., 2007) as their excitation contraction coupling changes to depend less on trans sarcolemmal $\mathrm{Ca}^{2+}$-transport and more on L-type $\mathrm{Ca}^{2+}$-influx to trigger $\mathrm{Ca}^{2+}$-release from the SR (Huang et al., 2008). During maturation the functional significance of cytosolic and Mi-CK increases (Hoerter et al., 1994). In light of the question whether CK facilitates transport across MOM, it is tempting to speculate that the increase in Mi-CK during development occurs simply because the cells transition from glycolytic to mitochondrial energy generation (Ostadal et al., 1999) and functional coupling of CK to an energy generation site is necessary for this reaction to happen in the direction of PCr synthesis. Probably, the concomitant decrease in apparent ADP-affinity is not for increasing energetic communication via the CK system, but due to the simultaneous development of the SR. The SR is less developed in lower vertebrates (Santer, 1985; Franzini-Armstrong and Boncompagni, 2011). In mammal heart, the SR develops significantly postnatally (Dan et al., 2007; Huang et al., 2008), and so does its association with mitochondria (Boncompagni et al., 2009). Thus, changes in SR-mitochondria interactions might provide an alternative explanation for the inter-species differences and developmental changes in the apparent ADP-affinity of permeabilized cardiomyocytes (Ventura-Clapier et al., 1998; Sokolova et al., 2009; Anmann et al., 2014). Additionally, the low
A

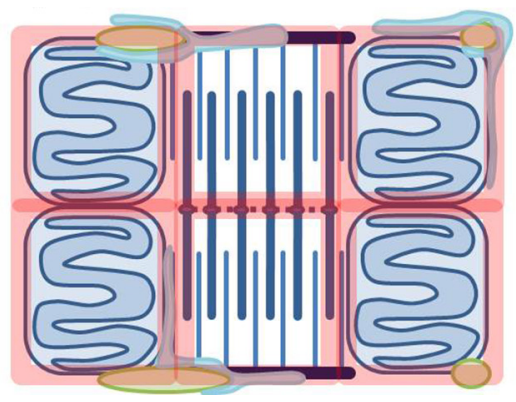

B

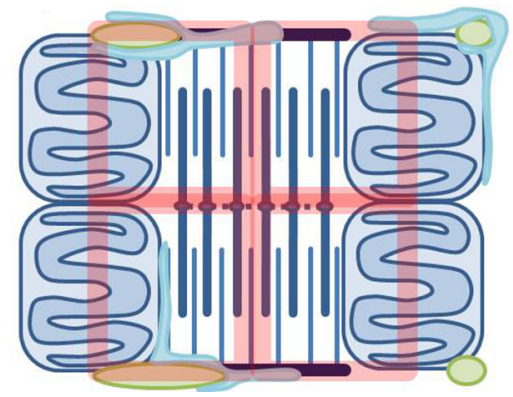

Mitochondrion

Sarcoplasmic reticulum

T-tubule

Z-line

-. M-band

Diffusion barrier
FIGURE 2 | Two scenarios for how diffusional barriers may be organized in cardiomyocytes. The schematic drawings are scaled according to Birkedal et al. (2006), Hayashi et al. (2009) and show mitochondria, t-tubules and sarcoplasmic reticulum (SR) around a sarcomere. The diffusional barriers are drawn to scale according to Illaste et al. (2012) and superimposed. In (A) the barriers are in agreement with the cell structures, but seem to separate mitochondria and myosin ATPases. In (B) mitochondria are grouped together with ATPases, but this scenario is difficult to explain in structural terms. 
apparent ADP-affinity is specific for oxidative muscles (Kuznetsov et al., 1996; Ventura-Clapier et al., 1998). The difference between muscles might be explained by distinct expression of tubulin that regulate VDAC gating (Varikmaa et al., 2014). Another explanation involves SR-mitochondria interaction that seems to be more prominent in muscles that are rich in mitochondria (FranziniArmstrong, 2007).

The close association of SR and mitochondria is crucial for proper function and energetic regulation. By ensuring mitochondrial $\mathrm{Ca}^{2+}$-uptake, it allows for regulation of mitochondrial energy production by $\mathrm{Ca}^{2+}$ as well as ADP and $\mathrm{P}_{\mathrm{i}}$. Moreover, it ensures sufficient energy supply to SERCA. Indeed, mitochondria are as efficient as CK in providing energy for SERCA function (Kaasik et al., 2001). Furthermore, as is the subject of this special issue, mitochondria are not only energetic but also redox hubs since they generate reactive oxygen species (ROS) with signaling potential. Mitochondrial $\mathrm{Ca}^{2+}$-uptake may take part in the regulation of ROS. As noted in the beginning, $\mathrm{Ca}^{2+}$ uptake by mitochondria increases NADH. Therefore, the $\mathrm{Ca}^{2+}$-uptake under physiological conditions may have similar effects as the addition of respiratory substrates: increase in $\mathrm{NAD}(\mathrm{P}) \mathrm{H}$ in turn used to reduce glutathion and thioredoxin pools, important antioxidant systems modulating mitochondrial ROS-emission (Garcia et al., 2010; Stanley et al., 2011; Aon et al., 2012). Compromised energy provision affects SERCA activity and $\mathrm{Ca}^{2+}$-re-uptake. This may lead to an increase in mitochondrial $\mathrm{Ca}^{2+}$, which is known to be an important trigger of the permeability transition pore (mPTP) (Bernardi, 1999). mPTP opening is to some extent reversible. But high enough $\mathrm{Ca}^{2+}$ and stimulation by other factors leads to irreversible opening, and eventually to apoptosis and/or necrosis (Di Lisa et al., 2011). Functional coupling between SR and mitochondria will ensure energy supply to SERCA thus preventing excessive mitochondrial $\mathrm{Ca}^{2+}$-uptake. In a situation of limited energy supply, transport of ADP and $\mathrm{P}_{\mathrm{i}}$ from SERCA to the mitochondria may increase the mitochondrial $\mathrm{Ca}^{2+}$-uptake capacity before mPTP opening is triggered (Wei et al., 2012; Sokolova et al., 2013).

\section{CONCLUDING REMARKS}

Feedback regulation of energetics depends on the location of diffusion barriers. We suggest an alternative explanation for the diffusion restriction at MOM level, namely that it is due to a close association of mitochondria and SR that ensures SERCA energy supply as well as mitochondrial regulation by $\mathrm{Ca}^{2+}$. It has been suggested that in cardiomyocytes, the permeability of MOM itself is regulated by tubulin. Indeed, "closing the gates" to the mitochondria to enhance energetic communication by the CK system seems like a good explanation, if it is more efficient. There are, however, some studies suggesting that a significant fraction of the energetic communication can occur as direct transport of ATP, ADP, and Pi. Also, cardiac function is not severely compromised by the lack of a functional CK system although diffusion restriction at the level of MOM is unchanged.

Whether the compartmentalization of energy units control the feedback exerted by the CK system or merely exists as part of a structural organization to optimize energy transfer between mitochondria and ATPases via ADP, $\mathrm{P}_{\mathrm{i}}$, and $\mathrm{Ca}^{2+}$, remain open questions.

\section{ACKNOWLEDGMENT}

The authors were funded by the European Union through the European Regional Development Fund.

\section{REFERENCES}

Akki, A., Su, J., Yano, T., Gupta, A., Wang, Y., Leppo, M. K., et al. (2012). Creatine kinase overexpression improves ATP kinetics and contractile function in postischemic myocardium. Am. J. Physiol. Heart Circ. Physiol. 303, H844-H852. doi: 10.1152/ajpheart.00268.2012

Anmann, T., Varikmaa, M., Timohhina, N., Tepp, K., Shevchuk, I., Chekulayev, V., et al. (2014). Formation of highly organized intracellular structure and energy metabolism in cardiac muscle cells during postnatal development of rat heart. Biochim. Biophys. Acta 1837, 1350-1361. doi: 10.1016/j.bbabio.2014.03.015

Aon, M. A., Cortassa, S., and O'Rourke, B. (2004). Percolation and criticality in a mitochondrial network. Proc. Natl. Acad. Sci. U.S.A. 101, 4447-4452. doi: 10.1073/pnas.0307156101

Aon, M. A., Stanley, B. A., Sivakumaran, V., Kembro, J. M., O’Rourke, B., Paolocci, N., et al. (2012). Glutathione/thioredoxin systems modulate mitochondrial $\mathrm{H}_{2} \mathrm{O}_{2}$ emission: an experimental-computational study. J. Gen. Physiol. 139, 479-491. doi: 10.1085/jgp.201210772

Arrio-Dupont, M. (1988). An example of substrate channeling between coimmobilized enzymes. Coupled activity of myosin ATPase and creatine kinase bound to frog heart myofilaments. FEBS Lett. 240, 181-185. doi: 10.1016/00145793(88)80364-8

Arrio-Dupont, M., Coulet, P. R., and Gautheron, D. C. (1985). Coupled reaction of immobilized aspartate aminotransferase and malate dehydrogenase. A plausible model for the cellular behaviour of these enzymes. Biochim. Biophys. Acta 829, 58-68. doi: 10.1016/0167-4838(85)90068-8

Balaban, R. S. (2002). Cardiac energy metabolism homeostasis: role of cytosolic calcium. J. Mol. Cell. Cardiol. 34, 1259-1271. doi: 10.1006/jmcc.2002.2082

Bernardi, P. (1999). Mitochondrial transport of cations: channels, exchangers, and permeability transition. Physiol. Rev. 79, 1127-1155.

Birkedal, R., and Gesser, H. (2006). Intracellular compartmentation of cardiac fibres from rainbow trout and Atlantic cod-a general design of heart cells. Biochim. Biophys. Acta 1757, 764-772. doi: 10.1016/j.bbabio.2006.04.022

Birkedal, R., Shiels, H. A., and Vendelin, M. (2006). Three-dimensional mitochondrial arrangement in ventricular myocytes: from chaos to order. Am. J. Physiol. Cell Physiol. 291, C1148-C1158. doi: 10.1152/ajpcell.00236.2006

Boehm, E. A., Radda, G. K., Tomlin, H., and Clark, J. F. (1996). The utilisation of creatine and its analogues by cytosolic and mitochondrial creatine kinase. Biochim. Biophys. Acta 1274, 119-128. doi: 10.1016/0005-2728(96) 00018-7

Boncompagni, S., Rossi, A. E., Micaroni, M., Beznoussenko, G. V., Polishchuk, R. S., Dirksen, R. T., et al. (2009). Mitochondria are linked to calcium stores in striated muscle by developmentally regulated tethering structures. Mol. Biol. Cell 20, 1058-1067. doi: 10.1091/mbc.E08-07-0783

Bose, S., French, S., Evans, F. J., Joubert, F., and Balaban, R. S. (2003). Metabolic network control of oxidative phosphorylation: multiple roles of inorganic phosphate. J. Biol. Chem. 278, 39155-39165. doi: 10.1074/jbc.M306409200

Bottomley, P. A., Panjrath, G. S., Lai, S., Hirsch, G. A., Wu, K., Najjar, S. S., et al. (2013). Metabolic rates of ATP transfer through creatine kinase (CK Flux) predict clinical heart failure events and death. Sci. Transl. Med. 5, 215re3. doi: 10.1126/scitranslmed.3007328

Brandes, R., and Bers, D. M. (2002). Simultaneous measurements of mitochondrial NADH and $\mathrm{Ca}(2+)$ during increased work in intact rat heart trabeculae. Biophys. J. 83, 587-604. doi: 10.1016/S0006-3495(02)75194-1

Branovets, J., Sepp, M., Kotlyarova, S., Jepihhina, N., Sokolova, N., Aksentijevic, D., et al. (2013). Unchanged mitochondrial organization and compartmentation of high-energy phosphates in creatine deficient GAMT-/- mouse heart. Am. J. Physiol. Heart Circ. Physiol. 305, H506-H520. doi: 10.1152/ajpheart.00919.2012

Cortassa, S., Aon, M. A., O’Rourke, B., Jacques, R., Tseng, H.-J., Marbán, E., et al. (2006). A computational model integrating electrophysiology, contraction, and mitochondrial bioenergetics in the ventricular myocyte. Biophys. J. 91, 1564-1589. doi: 10.1529/biophysj.105.076174 
Crawford, R. M., Ranki, H. J., Botting, C. H., Budas, G. R., and Jovanovic, A. (2002). Creatine kinase is physically associated with the cardiac ATP-sensitive $\mathrm{K}^{+}$channel in vivo. FASEB J. 16, 102-104. doi: 10.1096/fj.01-0466fje

Crozatier, B., Badoual, T., Boehm, E., Ennezat, P. V., Guenoun, T., Su, J., et al. (2002). Role of creatine kinase in cardiac excitation-contraction coupling: studies in creatine kinase-deficient mice. FASEB J. 16, 653-660. doi: 10.1096/fj.010652com

Dan, P., Lin, E., Huang, J., Biln, P., and Tibbits, G. F. (2007). Three-dimensional distribution of cardiac $\mathrm{Na}^{+}-\mathrm{Ca}^{2+}$ exchanger and ryanodine receptor during development. Biophys. J. 93, 2504-2518. doi: 10.1529/biophysj.107.104943

Decker, M. L., Behnke-Barclay, M., Cook, M. G., Lesch, M., and Decker, R. S. (1991). Morphometric evaluation of the contractile apparatus in primary cultures of rabbit cardiac myocytes. Circ. Res. 69, 86-94. doi: 10.1161/01.RES.69.1.86

Di Lisa, F., Carpi, A., Giorgio, V., and Bernardi, P. (2011). The mitochondrial permeability transition pore and cyclophilin D in cardioprotection. Biochim. Biophys. Acta 1813, 1316-1322. doi: 10.1016/j.bbamcr.2011.01.031

Fossel, E. T., and Hoefeler, H. (1987). A synthetic functional metabolic compartment. The role of propinquity in a linked pair of immobilized enzymes. Eur. J. Biochem. 170, 165-171. doi: 10.1111/j.1432-1033.1987.tb13682.x

Franzini-Armstrong, C. (2007). ER-mitochondria communication. How privileged? Physiology (Bethesda) 22, 261-268. doi: 10.1152/physiol. 00017.2007

Franzini-Armstrong, C., and Boncompagni, S. (2011). The evolution of the mitochondria-to-calcium release units relationship in vertebrate skeletal muscles. J. Biomed. Biotechnol. 2011:830573. doi: 10.1155/2011/830573

Garcia, J., Han, D., Sancheti, H., Yap, L.-P., Kaplowitz, N., and Cadenas, E. (2010). Regulation of mitochondrial glutathione redox status and protein glutathionylation by respiratory substrates. J. Biol. Chem. 285, 39646-39654. doi: 10.1074/jbc.M110.164160

Goldman, R., and Katchalski, E. P. (1971). Kinetic behavior of a two-enzyme membrane carrying out a consecutive set of reactions. J. Theor. Biol. 32, 243-257. doi: 10.1016/0022-5193(71)90163-9

Grosse, R., Spitzer, E., Kupriyanov, V. V., Saks, V. A., and Repke, K. R. (1980). Coordinate interplay between $\left(\mathrm{Na}^{+}+\mathrm{K}^{+}\right)$-ATPase and creatine phosphokinase optimizes $\left(\mathrm{Na}^{+} / \mathrm{K}^{+}\right)$-antiport across the membrane of vesicles formed from the plasma membrane of cardiac muscle cell. Biochim. Biophys. Acta 603, 142-156. doi: 10.1016/0005-2736(80)90397-1

Gudbjarnason, S., Mathes, P., and Ravens, K. G. (1970). Functional compartmentation of ATP and creatine phosphate in heart muscle. J. Mol. Cell. Cardiol. 1, 325-339. doi: 10.1016/0022-2828(70)90009-X

Gupta, A., Akki, A., Wang, Y., Leppo, M. K., Chacko, V. P., Foster, D. B., et al. (2012). Creatine kinase-mediated improvement of function in failing mouse hearts provides causal evidence the failing heart is energy starved. J. Clin. Invest. 122, 291-302. doi: 10.1172/JCI57426

Gustafson, L. A., and van Beek, J. H. (2002). Activation time of myocardial oxidative phosphorylation in creatine kinase and adenylate kinase knockout mice. Am. J. Physiol. Heart Circ. Physiol. 282, H2259-H2264. doi: 10.1152/ajpheart.00264.2001

Haagensen, L., Jensen, D. H., and Gesser, H. (2008). Dependence of myosin-ATPase on structure bound creatine kinase in cardiac myofibrils from rainbow trout and freshwater turtle. Comp. Biochem. Physiol. A Mol. Integr. Physiol. 150, 404-409. doi: 10.1016/j.cbpa.2008.04.604

Hayashi, T., Martone, M. E., Yu, Z., Thor, A., Doi, M., Holst, M. J., et al. (2009). Three-dimensional electron microscopy reveals new details of membrane systems for $\mathrm{Ca}^{2+}$ signaling in the heart. J. Cell Sci. 122, 1005. doi: $10.1242 /$ jcs. 028175

Hettling, H., and van Beek, J. H. (2011). Analyzing the functional properties of the creatine kinase system with multiscale "Sloppy" Modeling. PLoS Comput. Biol. 7:e1002130. doi: 10.1371/journal.pcbi.1002130

Hoerter, J. A., Kuznetsov, A., and Ventura-Clapier, R. (1991). Functional development of the creatine kinase system in perinatal rabbit heart. Circ. Res. 69, 665-676. doi: 10.1161/01.RES.69.3.665

Hoerter, J. A., Ventura-Clapier, R., and Kuznetsov, A. (1994). Compartmentation of creatine kinases during perinatal development of mammalian heart. Mol. Cell. Biochem. 133-134, 277-286. doi: 10.1007/BF01267960

Huang, J., Hove-Madsen, L., and Tibbits, G. F. (2008). Ontogeny of $\mathrm{Ca}^{2+}$-induced $\mathrm{Ca}^{2+}$ release in rabbit ventricular myocytes. Am. J. Physiol. Cell Physiol. 294, C516-C525. doi: 10.1152/ajpcell.00417.2007
Illaste, A., Laasmaa, M., Peterson, P., and Vendelin, M. (2012). Analysis of molecular movement reveals latticelike obstructions to diffusion in heart muscle cells. Biophys. J. 102, 739-748. doi: 10.1016/j.bpj.2012.01.012

Jepihhina, N., Beraud, N., Sepp, M., Birkedal, R., and Vendelin, M. (2011). Permeabilized rat cardiomyocyte response demonstrates intracellular origin of diffusion obstacles. Biophys. J. 101, 2112-2121. doi: 10.1016/j.bpj.2011. 09.025

Kaasik, A., Veksler, V., Boehm, E., Novotova, M., Minajeva, A., and VenturaClapier, R. (2001). Energetic crosstalk between organelles: architectural integration of energy production and utilization. Circ. Res. 89, 153-159. doi: 10.1161/hh1401.093440

Kan, H. E., Renema, W. K. J., Isbrandt, D., and Heerschap, A. (2004). Phosphorylated guanidinoacetate partly compensates for the lack of phosphocreatine in skeletal muscle of mice lacking guanidinoacetate methyltransferase. J. Physiol. 560, 219-229. doi: 10.1113/jphysiol.2004.067926

Karo, J., Peterson, P., and Vendelin, M. (2012). Molecular dynamics simulations of creatine kinase and adenine nucleotide translocase in mitochondrial membrane patch. J. Biol. Chem. 287, 7467-7476. doi: 10.1074/jbc.M111.332320

Katz, L. A., Swain, J. A., Portman, M. A., and Balaban, R. S. (1989). Relation between phosphate metabolites and oxygen consumption of heart in vivo. Am. J. Physiol. 256, H265-H274.

Kinsey, S. T., Locke, B. R., and Dillaman, R. M. (2011). Molecules in motion: influences of diffusion on metabolic structure and function in skeletal muscle. J. Exp. Biol. 214, 263-274. doi: 10.1242/jeb.047985

Kraft, T., Hornemann, T., Stolz, M., Nier, V., and Wallimann, T. (2000). Coupling of creatine kinase to glycolytic enzymes at the sarcomeric I-band of skeletal muscle: a biochemical study in situ. J. Muscle Res. Cell Motil. 21, 691-703. doi: 10.1023/A:1005623002979

Kritzer, M. D., Li, J., Dodge-Kafka, K., and Kapiloff, M. S. (2012). AKAPs: the architectural underpinnings of local cAMP signaling. J. Mol. Cell. Cardiol. 52, 351-358. doi: 10.1016/j.yjmcc.2011.05.002

Kummel, L. (1988). Ca, Mg-ATPase activity of permeabilised rat heart cells and its functional coupling to oxidative phosphorylation of the cells. Cardiovasc. Res. 22, 359-367. doi: 10.1093/cvr/22.5.359

Kuznetsov, A. V., Tiivel, T., Sikk, P., Kaambre, T., Kay, L., Daneshrad, Z., et al. (1996). Striking differences between the kinetics of regulation of respiration by ADP in slow-twitch and fast-twitch muscles in vivo. Eur. J. Biochem. 241, 909-915. doi: 10.1111/j.1432-1033.1996.00909.x

Laasmaa, M., Vendelin, M., and Peterson, P. (2011). Application of regularized Richardson-Lucy algorithm for deconvolution of confocal microscopy images. J. Microsc. 243, 124-140. doi: 10.1111/j.1365-2818.2011.03486.x

Lu, X., Ginsburg, K. S., Kettlewell, S., Bossuyt, J., Smith, G. L., and Bers, D. M. (2013). Measuring local gradients of intramitochondrial $\left[\mathrm{Ca}^{2+}\right]$ in cardiac myocytes during sarcoplasmic reticulum $\mathrm{Ca}^{2+}$ release. Circ. Res. 112, 424-431. doi: 10.1161/CIRCRESAHA.111.300501

Lygate, C. A., Aksentijevic, D., Dawson, D., ten Hove, M., Phillips, D., de Bono, J. P., et al. (2013). Living without creatine unchanged exercise capacity and response to chronic myocardial infarction in creatine-deficient mice. Circ. Res. 112, 945-955. doi: 10.1161/CIRCRESAHA.112.300725

Lygate, C. A., Hunyor, I., Medway, D., de Bono, J. P., Dawson, D., Wallis, J., et al. (2009). Cardiac phenotype of mitochondrial creatine kinase knockout mice is modified on a pure C57BL/6 genetic background. J. Mol. Cell. Cardiol. 46, 93-99. doi: 10.1016/j.yjmcc.2008.09.710

Lygate, C. A., Medway, D. J., Ostrowski, P. J., Aksentijevic, D., Sebag-Montefiore, L., Hunyor, I., et al. (2012). Chronic creatine kinase deficiency eventually leads to congestive heart failure, but severity is dependent on genetic background, gender and age. Basic Res. Cardiol. 107, 276-286. doi: 10.1007/s00395-0120276-2

Lygate, C. A., and Neubauer, S. (2014). Metabolic flux as a predictor of heart failure prognosis. Circ. Res. 114, 1228-1230. doi: 10.1161/CIRCRESAHA.114.303551

McCormack, J. G., Halestrap, A. P., and Denton, R. M. (1990). Role of calcium ions in regulation of mammalian intramitochondrial metabolism. Physiol. Rev. 70, 391-425.

Mekhfi, H., Hoerter, J., Lauer, C., Wisnewsky, C., Schwartz, K., and VenturaClapier, R. (1990). Myocardial adaptation to creatine deficiency in rats fed with beta- guanidinopropionic acid, a creatine analogue. Am. J. Physiol. 258, H1151-H1158.

Meyer, R. A., Sweeney, H. L., and Kushmerick, M. J. (1984). A simple analysis of the phosphocreatine shuttle. Am. J. Physiol. 246, C365-C377. 
Mika, D., Leroy, J., Vandecasteele, G., and Fischmeister, R. (2012). PDEs create local domains of cAMP signaling. J. Mol. Cell. Cardiol. 52, 323-329. doi: 10.1016/j.yjmcc.2011.08.016

Minajeva, A., Ventura-Clapier, R., and Veksler, V. (1996). $\mathrm{Ca}^{2+}$ uptake by cardiac sarcoplasmic reticulum ATPase in situ strongly depends on bound creatine kinase. Pflugers Arch. 432, 904-912. doi: 10.1007/s004240050214

Nahrendorf, M., Spindler, M., Hu, K., Bauer, L., Ritter, O., Nordbeck, P., et al. (2005). Creatine kinase knockout mice show left ventricular hypertrophy and dilatation, but unaltered remodeling post-myocardial infarction. Cardiovasc. Res. 65, 419-427. doi: 10.1016/j.cardiores.2004.10.006

Neubauer, S., Hu, K., Horn, M., Remkes, H., Hoffmann, K. D., Schmidt, C., et al. (1999). Functional and energetic consequences of chronic myocardial creatine depletion by beta-guanidinopropionate in perfused hearts and in intact rats. J. Mol. Cell. Cardiol. 31, 1845-1855. doi: 10.1006/jmcc.1999.1016

Ostadal, B., Ostadalova, I., and Dhalla, N. S. (1999). Development of cardiac sensitivity to oxygen deficiency: comparative and ontogenetic aspects. Physiol. Rev. 79, 635-659.

Ramay, H. R., and Vendelin, M. (2009). Diffusion restrictions surrounding mitochondria: a mathematical model of heart muscle fibers. Biophys. J. 97, 443-452. doi: 10.1016/j.bpj.2009.04.062

Rojo, M., Hovius, R., Demel, R., Wallimann, T., Eppenberger, H. M., and Nicolay, K. (1991). Interaction of mitochondrial creatine kinase with model membranes A monolayer study. FEBS Lett. 281, 123-129. doi: 10.1016/0014-5793(91) 80374-C

Rostovtseva, T., and Colombini, M. (1997). VDAC channels mediate and gate the flow of ATP: implications for the regulation of mitochondrial function. Biophys. J. 72, 1954-1962. doi: 10.1016/S0006-3495(97)78841-6

Rostovtseva, T. K., and Bezrukov, S. M. (2012). VDAC inhibition by tubulin and its physiological implications. Biochim. Biophys. Acta 1818, 1526-1535. doi: 10.1016/j.bbamem.2011.11.004

Rostovtseva, T. K., Sheldon, K. L., Hassanzadeh, E., Monge, C., Saks, V., Bezrukov, S. M., et al. (2008). Tubulin binding blocks mitochondrial voltage-dependent anion channel and regulates respiration. Proc. Natl. Acad. Sci. U.S.A. 105, 18746-18751. doi: 10.1073/pnas.0806303105

Saks, V. A., Belikova, Y. O., and Kuznetsov, A. V. (1991). In vivo regulation of mitochondrial respiration in cardiomyocytes: specific restrictions for intracellular diffusion of ADP. Biochim. Biophys. Acta 1074, 302-311. doi: 10.1016/03044165(91)90168-G

Saks, V. A., Kaambre, T., Sikk, P., Eimre, M., Orlova, E., Paju, K., et al. (2001). Intracellular energetic units in red muscle cells. Biochem. J. 356, 643-657. doi: 10.1042/0264-6021:3560643

Saks, V. A., Khuchua, Z. A., Vasilyeva, E. V., Belikova, Y. O., and Kuznetsov, A. V. (1994). Metabolic compartmentation and substrate channelling in muscle cells. Role of coupled creatine kinases in in vivo regulation of cellular respiration-a synthesis. Mol. Cell. Biochem. 133-134, 155-192. doi: 10.1007/BF01267954

Saks, V. A., Kongas, O., Vendelin, M., and Kay, L. (2000). Role of the creatine/phosphocreatine system in the regulation of mitochondrial respiration. Acta Physiol. Scand. 168, 635-641. doi: 10.1046/j.1365-201x.2000.00715.x

Saks, V. A., Vasil'eva, E., Belikova, Y. O., Kuznetsov, A. V., Lyapina, S., Petrova, L., et al. (1993). Retarded diffusion of ADP in cardiomyocytes: possible role of mitochondrial outer membrane and creatine kinase in cellular regulation of oxidative phosphorylation. Biochim. Biophys. Acta 1144, 134-148.

Santer, R. M. (1985). Morphology and innervation of the fish heart. Adv. Anat. Embryol. Cell Biol. 89, 1-102. doi: 10.1007/978-3-642-70135-1_1

Schlattner, U., Tokarska-Schlattner, M., Ramirez, S., Brückner, A., Kay, L., Polge, C., et al. (2009). Mitochondrial kinases and their molecular interaction with cardiolipin. Biochim. Biophys. Acta 1788, 2032-2047. doi: 10.1016/j.bbamem.2009.04.018

Schmidt, A., Marescau, B., Boehm, E. A., Renema, W. K., Peco, R., Das, A., et al. (2004). Severely altered guanidino compound levels, disturbed body weight homeostasis and impaired fertility in a mouse model of guanidinoacetate Nmethyltransferase (GAMT) deficiency. Hum. Mol. Genet. 13, 905-921. doi: $10.1093 / \mathrm{hmg} / \mathrm{ddh} 112$

Sedarat, F., Xu, L., Moore, E. D., and Tibbits, G. F. (2000). Colocalization of dihydropyridine and ryanodine receptors in neonate rabbit heart using confocal microscopy. Am. J. Physiol. Heart Circ. Physiol. 279, H202-H209.

Sepp, M., Sokolova, N., Jugai, S., Mandel, M., Peterson, P., and Vendelin, M. (2014). Tight coupling of $\mathrm{Na}^{+} / \mathrm{K}^{+}$-ATPase with glycolysis demonstrated in permeabilized rat cardiomyocytes. PLoS ONE 9:e99413. doi: 10.1371/journal.pone.0099413

Sepp, M., Vendelin, M., Vija, H., and Birkedal, R. (2010). ADP compartmentation analysis reveals coupling between pyruvate kinase and ATPases in heart muscle. Biophys. J. 98, 2785-2793. doi: 10.1016/j.bpj.2010.03.025

Seppet, E. K., Kaambre, T., Sikk, P., Tiivel, T., Vija, H., Tonkonogi, M., et al. (2001). Functional complexes of mitochondria with Ca,MgATPases of myofibrils and sarcoplasmic reticulum in muscle cells. Biochim. Biophys. Acta 1504, 379-395. doi: 10.1016/S0005-2728(00)00269-3

Shoubridge, E. A., Jeffry, F. M., Keogh, J. M., Radda, G. K., and Seymour, A. M. (1985). Creatine kinase kinetics, ATP turnover, and cardiac performance in hearts depleted of creatine with the substrate analogue beta- guanidinopropionic acid. Biochim. Biophys. Acta 847, 25-32. doi: 10.1016/0167-4889(85) 90148-X

Sokolova, N., Pan, S., Provazza, S., Beutner, G., Vendelin, M., Birkedal, R., et al. (2013). ADP protects cardiac mitochondria under severe oxidative stress. PLoS ONE 8:e83214. doi: 10.1371/journal.pone.0083214

Sokolova, N., Vendelin, M., and Birkedal, R. (2009). Intracellular diffusion restrictions in isolated cardiomyocytes from rainbow trout. BMC Cell Biol. 10:90. doi: 10.1186/1471-2121-10-90

Spindler, M., Meyer, K., Strömer, H., Leupold, A., Boehm, E., Wagner, H., et al. (2004). Creatine kinase-deficient hearts exhibit increased susceptibility to ischemia-reperfusion injury and impaired calcium homeostasis. Am. J. Physiol. Heart Circ. Physiol. 287, H1039-H1045. doi: 10.1152/ajpheart.01016.2003

Stanley, B. A., Sivakumaran, V., Shi, S., McDonald, I., Lloyd, D., Watson, W. H., et al. (2011). Thioredoxin reductase-2 is essential for keeping low levels of $\mathrm{H}_{2} \mathrm{O}_{2}$ emission from isolated heart mitochondria. J. Biol. Chem. 286, 33669-33677. doi: 10.1074/jbc.M111.284612

Territo, P. R., French, S. A., Dunleavy, M. C., Evans, F. J., and Balaban, R. S. (2001). Calcium activation of heart mitochondrial oxidative phosphorylation: rapid kinetics of mVO2, NADH, AND light scattering. J. Biol. Chem. 276, 2586-2599. doi: 10.1074/jbc.M002923200

Territo, P. R., Mootha, V. K., French, S. A., and Balaban, R. S. (2000). Ca(2+) activation of heart mitochondrial oxidative phosphorylation: role of the $\mathrm{F}(0) / \mathrm{F}(1)$ ATPase. Am. J. Physiol. Cell Physiol. 278, C423-C435.

Tiivel, T., Kadaya, L., Kuznetsov, A., Kaambre, T., Peet, N., Sikk, P., et al. (2000). Developmental changes in regulation of mitochondrial respiration by ADP and creatine in rat heart in vivo. Mol. Cell. Biochem. 208, 119-128. doi: 10.1023/A:1007002323492

Varikmaa, M., Bagur, R., Kaambre, T., Grichine, A., Timohhina, N., Tepp, K., et al. (2014). Role of mitochondria-cytoskeleton interactions in respiration regulation and mitochondrial organization in striated muscles. Biochim. Biophys. Acta 1837, 232-245. doi: 10.1016/j.bbabio.2013.10.011

Vendelin, M., Béraud, N., Guerrero, K., Andrienko, T., Kuznetsov, A. V., Olivares, J., et al. (2005). Mitochondrial regular arrangement in muscle cells: a "crystallike” pattern. Am. J. Physiol. Cell Physiol. 288, C757-C767. doi: 10.1152/ajpcell.00281.2004

Vendelin, M., and Birkedal, R. (2008). Anisotropic diffusion of fluorescently labeled ATP in rat cardiomyocytes determined by raster image correlation spectroscopy. Am. J. Physiol. Cell Physiol. 295, C1302-C1315. doi: 10.1152/ajpcell.00313.2008

Vendelin, M., Eimre, M., Seppet, E., Peet, N., Andrienko, T., Lemba, M., et al. (2004a). Intracellular diffusion of adenosine phosphates is locally restricted in cardiac muscle. Mol. Cell. Biochem. 256, 229-241. doi: 10.1023/B:MCBI.0000009871.04141.64

Vendelin, M., Lemba, M., and Saks, V. A. (2004b). Analysis of functional coupling: mitochondrial creatine kinase and adenine nucleotide translocase. Biophys. J. 87, 696-713. doi: 10.1529/biophysj.103.036210

Vendelin, M., Hoerter, J. A., Mateo, P., Soboll, S., Gillet, B., and Mazet, J.-L. (2010). Modulation of energy transfer pathways between mitochondria and myofibrils by changes in performance of perfused heart. J. Biol. Chem. 285, 37240-37250. doi: 10.1074/jbc.M110.147116

Ventura-Clapier, R., Kuznetsov, A., Veksler, V., Boehm, E., and Anflous, K. (1998). Functional coupling of creatine kinases in muscles: species and tissue specificity. Mol. Cell. Biochem. 184, 231-247. doi: 10.1023/A:1006840508139

Ventura-Clapier, R., Mekhfi, H., and Vassort, G. (1987). Role of creatine kinase in force development in chemically skinned rat cardiac muscle. J. Gen. Physiol. 89, 815-837. doi: 10.1085/jgp.89.5.815

Wallimann, T., Wyss, M., Brdiczka, D., Nicolay, K., and Eppenberger, H. M. (1992). Intracellular compartmentation, structure and function of creatine 
kinase isoenzymes in tissues with high and fluctuating energy demands: the "phosphocreatine circuit" for cellular energy homeostasis. Biochem. J. 281(Pt 1), 21-40.

Wang, S.-Q., Wei, C., Zhao, G., Brochet, D. X. P., Shen, J., Song, L.-S., et al. (2004). Imaging microdomain $\mathrm{Ca}^{2+}$ in muscle cells. Circ. Res. 94, 1011-1022. doi: 10.1161/01.RES.0000125883.68447.A1

Wei, A. C., Liu, T., Cortassa, S., Winslow, R. L., and O’Rourke, B. (2011). Mitochondrial $\mathrm{Ca}(2+)$ influx and efflux rates in guinea pig cardiac mitochondria:Low and high affinity effects of cyclosporine A. Biochim. Biophys. Acta. 1813, 1373-1381. doi: 10.1016/j.bbamcr.2011.02.012

Wei, A.-C., Liu, T., Winslow, R. L., and O’Rourke, B. (2012). Dynamics of matrixfree $\mathrm{Ca}^{2+}$ in cardiac mitochondria: two components of $\mathrm{Ca}^{2+}$ uptake and role of phosphate buffering. J. Gen. Physiol. 139, 465-478. doi: 10.1085/jgp. 201210784

Wu, F., and Beard, D. (2009). Roles of the creatine kinase system and myoglobin in maintaining energetic state in the working heart. BMC Syst. Biol. 3:22. doi: 10.1186/1752-0509-3-22

Wu, F., Zhang, E. Y., Zhang, J., Bache, R. J., and Beard, D. A. (2008). Phosphate metabolite concentrations and ATP hydrolysis potential in normal and ischaemic hearts. J. Physiol. 586, 4193-4208. doi: 10.1113/jphysiol.2008. 154732
Zweier, J. L., Jacobus, W. E., Korecky, B., and Brandejs-Barry, Y. (1991). Bioenergetic consequences of cardiac phosphocreatine depletion induced by creatine analogue feeding. J. Biol. Chem. 266, 20296-20304.

Conflict of Interest Statement: The authors declare that the research was conducted in the absence of any commercial or financial relationships that could be construed as a potential conflict of interest.

Received: 04 June 2014; accepted: 10 September 2014; published online: 29 September 2014.

Citation: Birkedal R, Laasmaa $M$ and Vendelin $M$ (2014) The location of energetic compartments affects energetic communication in cardiomyocytes. Front. Physiol. 5:376. doi: 10.3389/fphys.2014.00376

This article was submitted to Mitochondrial Research, a section of the journal Frontiers in Physiology.

Copyright (c) 2014 Birkedal, Laasmaa and Vendelin. This is an open-access article distributed under the terms of the Creative Commons Attribution License (CC BY). The use, distribution or reproduction in other forums is permitted, provided the original author(s) or licensor are credited and that the original publication in this journal is cited, in accordance with accepted academic practice. No use, distribution or reproduction is permitted which does not comply with these terms. 